\title{
Hua Measures on the Space of $p$-adic Matrices \\ and Inverse Limits of Grassmannians
}

\section{Yury Neretin}

Vienna, Preprint ESI 2367 (2012)

May 9, 2012

Supported by the Austrian Federal Ministry of Education, Science and Culture Available online at http://www.esi.ac.at 


\title{
Hua measures on the space of $p$-adic matrices and inverse limits of Grassmannians
}

\author{
YURY NERETIN ${ }^{1}$
}

We construct $p$-adic counterparts of Hua measures, inverse limits of $p$-adic Grassmannians, and describe natural groups of symmetries of such inverse limits.

\section{Results of the paper}

1.1. Real archetype. For details, see [8], [10], Section II.2.10. Denote by $\mathrm{U}(k)$ the group of unitary matrices of size $k$, by $d \chi^{k}$ the probability Haar measure on $\mathrm{U}(k)$. By $\mathrm{U}(\infty)$ we denote the inductive limit

$$
\ldots \longrightarrow \mathrm{U}(n) \longrightarrow \mathrm{U}(n+1) \longrightarrow \ldots
$$

of groups $\mathrm{U}(n)$.

Represent an element $g \in \mathrm{U}(n+1)$ as a block matrix $g=\left(\begin{array}{ll}a & b \\ c & d\end{array}\right)$ of size $n+1$. Consider the Livshits map $\Upsilon: \mathrm{U}(n+1) \rightarrow \mathrm{U}(n)$ given by

$$
\Upsilon\left(\begin{array}{ll}
a & b \\
c & d
\end{array}\right)=a-b(1+d)^{-1} c .
$$

This map commutes with left and right actions of $\mathrm{U}(n)$,

$$
\Upsilon\left(h_{1} g h_{2}\right)=h_{1} \Upsilon(g) h_{2}, \quad \text { where } h_{1}, h_{2} \in \mathrm{U}(n) .
$$

Therefore, the pushforward of the Haar measure $d \chi^{n+1}$ under the map $\Upsilon$ is the Haar measure $d \chi^{n}$. Therefore there exists the inverse limit $\mathfrak{U}(\infty)$ of the chain

$$
\ldots \longleftarrow \mathrm{U}(n) \longleftarrow \mathrm{U}(n+1) \longleftarrow \ldots
$$

equipped with a probability measure $d \chi^{\infty}$. The space $\mathfrak{U}(\infty)$ is not a group, but the unitary group $\mathrm{U}(\infty)$ acts on $\mathfrak{U}(\infty)$ by left and right multiplication, thus we get a measure preserving action of $\mathrm{U}(\infty) \times \mathrm{U}(\infty)$ on $\mathfrak{U}(\infty)$.

More generally, we fix $\lambda \in \mathbb{C}$ and consider the probability measure

$$
\chi_{\lambda}^{n}=\prod_{k=1}^{n} \frac{\Gamma(k+\lambda) \Gamma(k+\bar{\lambda})}{\Gamma(k) \Gamma(k+\lambda+\bar{\lambda})} \operatorname{det}(1+g)^{\lambda} \operatorname{det}(1+\bar{g})^{\bar{\lambda}} d \chi^{n}(g)
$$

on $\mathrm{U}(n)$. This system of measures also is projective and we get a family of measures $\chi_{\lambda}^{\infty}$ on the inverse limit of the chain (1.2).

Applying the Cayley transform to matrices $g \in \mathrm{U}(n)$, we get the space of $n \times n$ Hermitian matrices and the measures of the form

$$
C(\lambda, n) \operatorname{det}(1+i X)^{-\lambda-n} \cdot \operatorname{det}(1-i X)^{-\bar{\lambda}-n} d X .
$$

${ }^{1}$ Supported by grant FWF, P22122. 
Total integrals for such measures (for real $\lambda$ ) were firstly evaluated by Hua Loo Keng [2].

Now there is a substantial harmonic analysis on $\mathfrak{U}(\infty)$, see [15], [1]. Similar inverse limits exists for all 10 series of compact semisimple symmetric spaces,

$$
\ldots \longleftarrow G(n) / K(n) \longleftarrow G(n+1) / K(n+1) \longleftarrow \ldots,
$$

the group $G(\infty)$ acts on the inverse limit, see [18], [8]. In [8] the group spaces $K \times K / K$, where $K=\mathrm{SO}(n), \mathrm{U}(n), \operatorname{Sp}(n)$ were considered. The Livshits map (1.1) sends symmetric matrices $\left(g=g^{t}\right)$ to symmetric, the space $\mathrm{U}(n) / \mathrm{O}(n)$ of unitary symmetric matrices can be identified with the real Lagrangian Grassmannian. Therefore we get a chain of Lagrangian Grassmannians

$$
\ldots \longleftarrow \mathrm{U}(n) / \mathrm{O}(n) \longleftarrow \mathrm{U}(n+1) / \mathrm{O}(n+1) \longleftarrow \ldots,
$$

see [10], Section 3.6. Other classical symmetric spaces can be reduced to the group case by the same trick (we choose an appropriate involution in a group manifold commuting with the map $\Upsilon$ ).

There is also a similar construction for symmetric group (see [3]), the limit object admits a substantial harmonic analysis. Our purpose is to obtain a $p$-adic counterpart of these constructions.

\subsection{Notation. Let}

- $\mathbb{Q}_{p}$ be the $p$-adic field;

- $\mathbb{O}_{p}$ the ring of $p$-adic integers;

- $\operatorname{vol}(\cdot)$ be the translation invariant $\sigma$-finite measure on a linear space $\mathbb{Q}_{p}^{m}$ normalized by the condition $\operatorname{vol}\left(\mathbb{O}_{p}^{m}\right)=1$;

- $|\cdot|$ be the norm on $\mathbb{Q}_{p}$

- $\operatorname{GL}\left(n, \mathbb{Q}_{p}\right)$ and $\operatorname{GL}\left(n, \mathbb{O}_{p}\right)$ be the groups of invertible $n \times n$ matrices over $\mathbb{Q}_{p}$ and $\mathbb{O}_{p}$

- $\operatorname{Mat}\left(n, \mathbb{Q}_{p}\right), \operatorname{Mat}\left(n, \mathbb{O}_{p}\right)$ be the spaces of all $n \times n$ matrices over $\mathbb{Q}_{p}$ and $\mathbb{O}_{p}$

- $\operatorname{Symm}\left(n, \mathbb{Q}_{p}\right), \operatorname{ASymm}\left(n, \mathbb{Q}_{p}\right)$ be spaces of symmetric (skew-symmetric matrices) over $\mathbb{Q}_{p}$;

- $g^{t}$ be transposed matrix;

- $\mathrm{Gr}_{2 n}^{n}$ is the Grassmannian of $n$-dimensional subspaces in $\mathbb{Q}_{p}^{2 n}$.

1.3. Measures $\mu_{s}^{n}$. Any $z \in \operatorname{Mat}\left(n, \mathbb{Q}_{p}\right)$ can be represented in the form

$$
z=A\left(\begin{array}{ccc}
p^{-k_{1}} & 0 & \ldots \\
0 & p^{-k_{2}} & \ldots \\
\vdots & \vdots & \ddots
\end{array}\right) B
$$

where $A, B \in \mathrm{GL}\left(n, \mathbb{O}_{p}\right)$ and

$$
k_{1} \geqslant k_{2} \geqslant \ldots \geqslant k_{n} \geqslant-\infty
$$

We say that $p^{k_{j}}$ are singular numbers of the matrix $z$. 
We define the function $\gamma(z)$ on $\operatorname{Mat}\left(n, \mathbb{O}_{p}\right)$ by

$$
\gamma(z)=\prod_{k_{j}>0} p^{k_{j}}
$$

\section{Theorem 1.1}

$$
\int_{\operatorname{Mat}\left(n, \mathbb{Q}_{p}\right)} \gamma(z)^{-\alpha} d \operatorname{vol}(z)=\prod_{j=1}^{n} \frac{1-p^{-\alpha+n-j}}{1-p^{-\alpha+n+j-1}}=: c(n, \alpha) .
$$

The integral converges if $\alpha>2 n-1$.

We define a measure $d \mu_{s}^{n}$, where $s>-1$, on $\operatorname{Mat}\left(n, \mathbb{Q}_{p}\right)$ by

$$
d \mu_{s}^{n}(z):=c(n, s+2 n)^{-1} \gamma(z)^{-s-2 n} d \operatorname{vol}_{n}(z) .
$$

The group $\operatorname{GL}\left(2 n, \mathbb{O}_{p}\right)$ acts on $\operatorname{Mat}\left(n, \mathbb{Q}_{p}\right)$ by linear-fractional transformations

$$
z \mapsto(a+z c)^{-1}(b+z d),
$$

where $\left(\begin{array}{ll}a & b \\ c & d\end{array}\right) \in \operatorname{GL}\left(2 n, \mathbb{O}_{p}\right)$ is a block $(n+n) \times(n+n)$ matrix. Notice, that this formula corresponds to the action of $\mathrm{GL}\left(2 n, \mathbb{O}_{p}\right)$ on the Grassmannian $\mathrm{Gr}_{2 n}^{n}$. Indeed, for an operator $z: \mathbb{Q}_{p}^{n} \rightarrow \mathbb{Q}_{p}^{n}$ consider its graph in $\mathbb{Q}_{p}^{n} \oplus \mathbb{Q}_{p}^{n}$, it consists of vectors

$$
v \oplus v z \text {, where } v \in \mathbb{Q}_{p}^{n} \text { is a row matrix. }
$$

The get a chart on Grassmannian, the complement of the chart has zero measure. A verification of (1.4) is straightforward (see, e.g., [10], Theorem 2.3.1).

Theorem 1.2 a) For any $\left(\begin{array}{ll}a & b \\ c & d\end{array}\right) \in \operatorname{GL}\left(2 n, \mathbb{Q}_{p}\right)$,

$$
d \mu_{s}^{n}\left((a+z c)^{-1}(b+z d)\right)=|\operatorname{det}(a+z c)|^{s} d \mu_{s}^{n}(z) .
$$

b) The measure $\mu_{s}^{n}$ is a unique probability Borel measure on $\operatorname{Mat}\left(n, \mathbb{Q}_{p}\right)$ satisfying the equation

$$
d \nu\left((a+z c)^{-1}(b+z d)\right)=|\operatorname{det}(a+z c)|^{s} d \nu(z)
$$

for any $\left(\begin{array}{ll}a & b \\ c & d\end{array}\right) \in \mathrm{GL}\left(2 n, \mathbb{O}_{p}\right)$.

In particular, for $s=0$ we get a unique $\mathrm{GL}\left(2 n, \mathbb{Q}_{p}\right)$-invariant measure on the Grassmannian $\mathrm{Gr}_{2 n}^{n}$.

1.4. Projective limits. Consider a $(n+1) \times(n+1)$ matrix

$$
z=\left(\begin{array}{ll}
z_{11} & z_{12} \\
z_{21} & z_{22}
\end{array}\right) .
$$


Consider the map $\Pi: \operatorname{Mat}\left(n+1, \mathbb{Q}_{p}\right) \rightarrow \operatorname{Mat}\left(n, \mathbb{Q}_{p}\right)$ given by

$$
\Pi:\left(\begin{array}{ll}
z_{11} & z_{12} \\
z_{21} & z_{22}
\end{array}\right) \mapsto z_{11} .
$$

Theorem 1.3 The pushforward of the measure $\mu_{s}^{n+1}$ under the map $\Pi$ is $\mu_{s}^{n}$

Thus we get a chain

$$
\ldots \longleftarrow\left(\operatorname{Mat}\left(n, \mathbb{Q}_{p}\right), d \mu_{s}^{n}\right) \longleftarrow\left(\operatorname{Mat}\left(n+1, \mathbb{Q}_{p}\right), d \mu_{s}^{n+1}\right) \longleftarrow \ldots
$$

By the Kolmogorov theorem (see, e.g. [20], Section 2.3, Theorem 3) the inverse limit in the category of measure spaces is well defined, denote by $d \mu_{s}^{\infty}$ the inverse limit of measures $\mu_{s}^{n}$, this measure can be regarded as a measure on $\operatorname{Mat}\left(\infty, \mathbb{Q}_{p}\right) \simeq \mathbb{Q}_{p}^{\infty \times \infty}$.

1.5. Symmetries of the measures $\mu_{s}^{\infty}$. Consider the chain of groups

$$
\cdots \longrightarrow \mathrm{GL}\left(2 n, \mathbb{O}_{p}\right) \longrightarrow \mathrm{GL}\left(2(n+1), \mathbb{O}_{p}\right) \longrightarrow \ldots
$$

and its inductive limit $\mathrm{GL}\left(2 \infty, \mathbb{O}_{p}\right)$. In other words, $\mathrm{GL}\left(2 \infty, \mathbb{O}_{p}\right)$ is the group of $(\infty+\infty) \times(\infty+\infty)$ matrices $g$ with integer elements such that $g^{-1}$ also has integer elements and $g-1$ has only finite number of non-zero entries.

Proposition 1.4 a) The measure $\mu_{s}$ is quasiinvariant with respect to the action $z \mapsto(a+z c)^{-1}(b+z d)$ of $\mathrm{GL}\left(2 \infty, \mathbb{O}_{p}\right)$. The Radon-Nikodym derivative is $|\operatorname{det}(a+z c)|^{s}$.

b) In particular for $s=0$ the measure $\mu_{s}^{\infty}$ is $\mathrm{GL}\left(2 \infty, \mathbb{Q}_{p}\right)$-invariant.

c) The measure $\mu_{s}^{\infty}$ is invariant with respect to the subgroup $P \subset \mathrm{GL}\left(2 \infty, \mathbb{Q}_{p}\right)$ consisting of matrices $\left(\begin{array}{ll}a & b \\ 0 & d\end{array}\right)$.

We must explain the meaning of the expression $|\operatorname{det}(a+z c)|^{s}$. Represent $g \in \mathrm{GL}\left(2 \infty, \mathbb{O}_{p}\right)$ as a block matrix of size $(k+\infty+k+\infty)$, where $k$ is sufficiently large,

$$
g=\left(\begin{array}{ll}
a & b \\
c & d
\end{array}\right)=\left(\begin{array}{cccc}
a_{11} & 0 & b_{11} & 0 \\
0 & 1 & 0 & 0 \\
c_{11} & 0 & d_{11} & 0 \\
0 & 0 & 0 & 1
\end{array}\right) .
$$

Represent $z \in \operatorname{Mat}\left(\infty, \mathbb{Q}_{p}\right)$ as a block matrix of size $(k+\infty)$ :

$$
z=\left(\begin{array}{ll}
z_{11} & z_{12} \\
z_{21} & z_{22}
\end{array}\right)
$$

Then

$$
\begin{aligned}
|\operatorname{det}(a+z c)|=\left|\operatorname{det}\left(\begin{array}{cc}
a_{11} & 0 \\
0 & 1
\end{array}\right)+\left(\begin{array}{cc}
z_{11} & z_{12} \\
z_{21} & z_{22}
\end{array}\right)\left(\begin{array}{cc}
c_{11} & 0 \\
0 & 0
\end{array}\right)\right|= \\
=\left|\operatorname{det}\left(\begin{array}{cc}
a_{11}+z_{11} c_{11} & 0 \\
z_{12} c_{11} & 1
\end{array}\right)\right|=\left|\operatorname{det}\left(a_{11}+z_{11} c_{11}\right)\right|,
\end{aligned}
$$


and we get a determinant of a finite matrix.

Next, we define two completions

$$
\overline{\overline{\mathrm{GL}}}\left(2 \infty, \mathbb{O}_{p}\right) \supset \overline{\mathrm{GL}}\left(2 \infty, \mathbb{O}_{p}\right) \supset \mathrm{GL}\left(2 \infty, \mathbb{O}_{p}\right)
$$

of the group $\mathrm{GL}\left(2 \infty, \mathbb{O}_{p}\right)$.

First, consider the group $\overline{\bar{T}}$ consisting of $(\infty+\infty) \times(\infty+\infty)$ matrices over $\mathbb{O}_{p}$ having the form

$$
h=\left(\begin{array}{ll}
a & b \\
0 & d
\end{array}\right),
$$

where

$$
a=\left(\begin{array}{cccc}
\alpha_{11} & 0 & 0 & \ldots \\
\alpha_{21} & \alpha_{22} & 0 & \ldots \\
\alpha_{31} & \alpha_{32} & \alpha_{33} & \ldots \\
\vdots & \vdots & \vdots & \ddots
\end{array}\right), \quad d=\left(\begin{array}{cccc}
\delta_{11} & \delta_{12} & \delta_{13} & \ldots \\
0 & \delta_{22} & \delta_{23} & \ldots \\
0 & 0 & \delta_{33} & \ldots \\
\vdots & \vdots & \vdots & \ddots
\end{array}\right),
$$

and

$$
\left|\alpha_{11}\right|=\left|\alpha_{22}\right|=\cdots=1 \quad\left|\delta_{11}\right|=\left|\delta_{22}\right|=\cdots=1 .
$$

As a set the group $\overline{\bar{T}}$ is a direct product of countable number of copies of $\mathbb{O}_{p} \backslash p \mathbb{O}_{p}$ (corresponding to $\alpha_{j j}$ and $\delta_{k k}$ ) and countable number of copies of $\mathbb{O}_{p}$ (corresponding to remaining $\alpha_{i j}, \delta_{k l}$, and to $\beta_{m n}$ ).

Also consider a smaller group $\bar{T} \subset \overline{\bar{T}}$ consisting of the matrices having the form (1.8)-(1.9) but (1.10) is replaced by

$$
\alpha_{11}=\alpha_{22}=\cdots=1 \quad \delta_{11}=\delta_{22}=\cdots=1 .
$$

REMARK. Notice that the matrices $h \in \overline{\bar{T}}$ can be made upper triangular after a permutation of basis elements (the first $\infty$ of basis elements must be written in the inverse order).

Denote by $\overline{\overline{\mathrm{GL}}}\left(2 \infty, \mathbb{O}_{p}\right)$ the group of matrices generated by $\mathrm{GL}\left(2 \infty, \mathbb{O}_{p}\right)$ and $\overline{\bar{T}}$. This group consists of invertible matrices $g=\left(\begin{array}{ll}a & b \\ c & d\end{array}\right)$ over $\mathbb{O}_{p}$ such that

$1^{\circ} . c$ has only finite number nonzero entries;

$2^{\circ}$. $a$ has only finite number of nonzero entries upper the diagonal;

$3^{\circ}$. $d$ has only finite number of nonzero entries lower the diagonal;

$4^{\circ} . g^{-1}$ has integer elements.

Denote by $\overline{\mathrm{GL}}\left(2 \infty, \mathbb{O}_{p}\right) \subset \overline{\overline{\mathrm{GL}}}\left(2 \infty, \mathbb{O}_{p}\right)$ the group of matrices generated by $\operatorname{GL}\left(2 \infty, \mathbb{O}_{p}\right)$ and $\bar{T}$. We must replace $2^{\circ}$ and $3^{\circ}$ by:

$2^{\circ}$. $a-1$ has only finite number of nonzero entries on the diagonal and upper the diagonal;

$3^{\circ \circ} \cdot d-1$ has only finite number of nonzero entries on the diagonal and lower the diagonal. 
Theorem 1.5 The group $\overline{\overline{\mathrm{GL}}}\left(2 \infty, \mathbb{O}_{p}\right)$ acts on $\operatorname{Mat}\left(\infty, \mathbb{Q}_{p}\right)$ by transformations $z \mapsto(a+z c)^{-1}(b+z d)$ leaving the measure $\mu_{s}^{\infty}$ quasiinvariant. The RadonNikodym derivative is $|\operatorname{det}(a+z c)|^{s}$.

The meaning of the expressions $(a+z c)^{-1}(b+z d)$ and $|\operatorname{det}(a+z c)|^{s}$ will be explained in Subsection 4.3.

Proposition 1.6 For any $g \in \overline{\mathrm{GL}}\left(2 \infty, \mathbb{O}_{p}\right)$ and $z \in \operatorname{Mat}\left(\infty, \mathbb{Q}_{p}\right)$, the expression

$$
\operatorname{det}(a+z c) \in \mathbb{Q}_{p}
$$

is well defined.

Denote by $\mathbb{Q}_{p}^{\times}, \mathbb{C}^{\times}$the multiplicative groups of $\mathbb{Q}_{p}$ and $\mathbb{C}$. Let $\chi$ be a homomorphism $\mathbb{Q}_{p}^{\times} \rightarrow \mathbb{C}^{\times},|\chi(z)|=1$. Then we can define a unitary representation of $\overline{\mathrm{GL}}\left(2 \infty, \mathbb{O}_{p}\right)$ in $L^{2}\left(\operatorname{Mat}\left(\infty, \mathbb{Q}_{p}\right), \mu_{s}^{\infty}\right)$ by the formula

$$
\rho_{s, \chi}\left(\begin{array}{ll}
a & b \\
c & d
\end{array}\right) f(z)=f\left((a+z c)^{-1}(b+z d)\right) \operatorname{det}(a+z c)^{s / 2} \chi(a+z c) .
$$

1.6. Some remarks on real-p-adic parallel. Analogs of noncompact Riemannian symmetric spaces over $p$-adic numbers are Bruhat-Tits buildings (here there is a well-known and deep parallel, see a discussion and further references in [10]). Analogs of Hua integrals for buildings exist (see [9]) and they are used below in Section 2. But it seems that they do not admit projective limits (moreover, real-p-adic analogy does not requires this, for noncompact Riemannian symmetric spaces there are no inverse limits, see [17]).

Apparently, there are no reasonable $p$-adic analogs of compact Riemannian symmetric spaces.

On the other hand, the classical compact Riemannian symmetric spaces are Grassmannians or isotropic Grassmannians (see, e.g., [10], Subsections D.1), our construction is an emulation of Pickrell's approach [18].

1.7. Other inverse limits of Grassmannians.

a) Symplectic Lagrangian Grassmannian. Consider the group $\operatorname{Sp}\left(2 n, \mathbb{O}_{p}\right)$ consisting of integer $(n+n) \times(n+n)$ matrices $\left(\begin{array}{ll}a & b \\ c & d\end{array}\right)$ preserving skew-symmetric bilinear form $\left(\begin{array}{cc}0 & 1 \\ -1 & 0\end{array}\right)$ in $\mathbb{Q}_{p}^{n} \oplus \mathbb{Q}_{p}^{n}$. Consider the Grassmannian $\mathrm{L}_{n}$ of Lagrangian subspaces in $\mathbb{Q}_{p}^{2 n}$. Almost all elements of $\mathrm{L}_{n}$ can be represented as graphs of operators $\mathbb{Q}_{p}^{n} \oplus 0 \rightarrow 0 \oplus \mathbb{Q}_{p}^{n}$, the corresponding matrices $z$ are symmetric (see, e.g., [10], Theorem 3.1.4). The action of the group $\operatorname{Sp}\left(2 n, \mathbb{O}_{p}\right)$ on $\operatorname{Symm}\left(n, \mathbb{Q}_{p}\right)$ is given by the same formula (1.4).

We define measures

$$
d \mu_{s}^{n}(z)=\gamma(z)^{-s-n-1} d \operatorname{vol}_{n}(z)
$$


on $\operatorname{Symm}\left(n, \mathbb{Q}_{p}\right)$, we choose normalizing constants $c(s, n)$ to obtain probability measures. Their Radon-Nikodym derivatives with respect to transformations in $\operatorname{Sp}\left(2 n, \mathbb{O}_{p}\right)$ are

$$
\frac{d \mu_{s}^{n}\left((a+z c)^{-1}(b+z d)\right)}{d \mu_{s}^{n}(z)}=|\operatorname{det}(a+z c)|^{s} .
$$

Next, these measures form a projective system with respect to the map $\Pi: \operatorname{Symm}\left(n+1, \mathbb{Q}_{p}\right) \rightarrow \operatorname{Symm}\left(n, \mathbb{Q}_{p}\right)$, see $(1.7)$.

Now we can consider the inverse limit of measure spaces

$$
\ldots \longleftarrow\left(\operatorname{Symm}\left(n, \mathbb{Q}_{p}\right), d \mu_{s}^{n}\right) \longleftarrow\left(\operatorname{Symm}\left(n+1, \mathbb{Q}_{p}\right), d \mu_{s}^{n+1}\right) \longleftarrow \ldots
$$

b) Isotropic orthogonal Grassmannians. Consider the group $\mathrm{O}\left(2 n, \mathbb{O}_{p}\right)$ consisting of integer $(n+n) \times(n+n)$ matrices $\left(\begin{array}{ll}a & b \\ c & d\end{array}\right)$ preserving the symmetric bilinear form $\left(\begin{array}{ll}0 & 1 \\ 1 & 0\end{array}\right)$ in $\mathbb{Q}_{p}^{n} \oplus \mathbb{Q}_{p}^{n}$. Consider the subgroup $\mathrm{SO}\left(2 n, \mathbb{O}_{p}\right) \subset \mathrm{O}\left(2 n, \mathbb{O}_{p}\right)$ consisting of matrices with determinant $=1$. Consider the Grassmasnnian $\mathrm{Is}_{n}$ of $n$-dimensional isotropic subspaces. The group $\mathrm{SO}\left(2 n, \mathbb{O}_{p}\right)$ has two orbits on $\mathrm{Is}_{n}$, we choose one. Namely, consider the set $\mathrm{Is}_{n}^{0}$ of $n$-dimensional isotropic subspaces $M$ such that $M \cap\left(0 \oplus \mathbb{Q}_{p}^{n}\right)$ has even dimension, see, e.g., [7], Proposition 2.2.2, Lemma 3.3.1. Almost all elements of $\mathrm{Is}_{n}^{0}$ are graph of operators $z: \mathbb{Q}_{p}^{n} \oplus 0 \rightarrow 0 \oplus \mathbb{Q}_{p}^{n}$ and matrices $z$ are skew-symmetric $\left(z=-z^{t}\right)$. We define probability measures

$$
d \mu_{s}^{n}(z)=a(s, n) \gamma(z)^{-s-n+1} d \operatorname{vol}_{n}(z) .
$$

on $\operatorname{ASymm}\left(n, \mathbb{Q}_{p}\right)$. Their Radon-Nikodym derivatives are given by the same formula (1.14). Measures $d \mu_{s}^{n}$ form a projective system with respect to the maps $\Pi: \operatorname{ASymm}\left(n+1, \mathbb{Q}_{p}\right) \rightarrow \operatorname{ASymm}\left(n, \mathbb{Q}_{p}\right)$. Again, we can consider inverse limits of measure spaces

$$
\ldots \longleftarrow\left(\operatorname{ASymm}\left(n, \mathbb{Q}_{p}\right), d \mu_{s}^{n}\right) \longleftarrow\left(\operatorname{ASymm}\left(n+1, \mathbb{Q}_{p}, d \mu_{s}^{n+1}\right), \longleftarrow \ldots\right.
$$

c) In both cases (symplectic and orthogonal), the situation is parallel to the picture described above.

But the author does not know the explicit analog of the formula (1.3) for complete measure, because our prove is a reduction to the beta-function of Bruhat-Tits buildings [9], which was evaluated only for $\operatorname{GL}\left(n, \mathbb{Q}_{p}\right)$-case. Also, we can normalize the measures (1.13), (1.15) only if they are finite. Evidently, this holds for $s=0$ since in this case we have measure on Grassmannians invariant with respect to transitive actions compact groups $\left(\operatorname{Sp}\left(2 n, \mathbb{O}_{p}\right)\right.$ and $\left.\operatorname{SO}\left(2 n, \mathbb{O}_{p}\right)\right)$. Since $\gamma(z)^{-\alpha}$ decreases as a function $\alpha$, we get that our measures are well-defined at least for $s \geqslant 0$.

1.8. Remark. On representations of infinite-dimensional classical $p$-adic groups. Basic representation theory of infinite-dimensional classical 
groups and infinite symmetric groups was developed in 70-80s, see [21], [22], [13] for symmetric groups and [12], [14], [16], [19], [16], [7] for classical groups. These works had various continuations, see, e.g., [7], [1], [15], [3], and further references in [11].

Representations of infinite-dimensional classical $p$-adic groups remains to be a non well-understood topic. Now two substantial constructions are known. The first is the Weil representation of infinite-dimensional symplectic group and the corresponding contractive semigroup $\left(\right.$ Nazarov $^{2}$, [6], [5], and a partial exposition in [10], Sections $10.7,11.2)$. The second is the multiplication of double cosets and a $p$-adic analog of characteristic operator-function, see [11].

For infinite-dimensional groups over finite fields, see [23].

1.9. Further structure of the paper. In Section 2 we evaluate the integral (1.3). In Section 3 we prove Theorem 1.5 on transformations of measures $\mu_{s}^{n}$. In Section 4 we prove the statements about measures $\mu_{s}^{\infty}$.

\section{Proofs. Calculation of the integral}

Here we prove Theorem 1.1. In this section,

$$
G:=\operatorname{GL}\left(n, \mathbb{Q}_{p}\right), \quad K:=\operatorname{GL}\left(n, \mathbb{O}_{p}\right) .
$$

Denote by Lat $(n)$ the set of lattices (see, e.g., [10], Section 10.3) in $\mathbb{Q}_{p}^{n}$, we have

$$
\operatorname{Lat}(n) \simeq G / K
$$

2.1. Properties of the function $\gamma$. The following statement is obvious.

Lemma 2.1 Let $z \in \operatorname{Mat}\left(n, \mathbb{Q}_{p}\right)$, $\operatorname{det}(z) \neq 0$. Then

$$
\begin{aligned}
\frac{\gamma(z)}{\gamma\left(z^{-1}\right)} & =|\operatorname{det}(z)| ; \\
\gamma(z) & =\operatorname{vol}\left(z \mathbb{O}_{p}^{n}+\mathbb{O}_{p}^{n}\right) ; \\
\gamma\left(z^{-1}\right)^{-1} & =\operatorname{vol}\left(z \mathbb{O}_{p}^{n} \cap \mathbb{O}_{p}^{n}\right) .
\end{aligned}
$$

Also, note that

$$
|\operatorname{det}(z)|=\operatorname{vol}\left(z \mathbb{O}_{p}^{n}\right)
$$

2.2. Haar measure. According [4], Section V.2, the Haar measure on $G$ is given by

$$
|\operatorname{det}(z)|^{-n} d \operatorname{vol}(z)
$$

It is convenient to normalize this measure as

$$
d \chi(z)=\frac{1}{\operatorname{vol}(K)}|\operatorname{det}(z)|^{-n} d \operatorname{vol}(z),
$$

then $\chi(K)=1$.

\footnotetext{
${ }^{2} \mathrm{~A}$ weaker version of construction is in [24].
} 


\section{Lemma 2.2}

$$
\operatorname{vol}(K)=\prod_{j=1}^{n}\left(1-p^{-j}\right)
$$

Proof. Consider the natural map $\operatorname{Mat}\left(n, \mathbb{O}_{p}\right) \rightarrow \operatorname{Mat}\left(n, \mathbb{F}_{p}\right)$, where $\mathbb{F}_{p}=$ $\mathbb{O}_{p} / p \mathbb{O}_{p}$ is the field with $p$ elements. The total number of points in $\operatorname{Mat}\left(n, \mathbb{F}_{p}\right)$ is $p^{n^{2}}$. The total number of points in $\operatorname{GL}\left(n, \mathbb{F}_{p}\right) \subset \operatorname{Mat}\left(n, \mathbb{F}_{p}\right)$ is $\prod_{j=1}^{n}\left(p^{n}-p^{j}\right)$.

2.3. Calculation of the integral. Keeping in the mind the expression for Haar measure we transform our integral (1.3) as

$$
\begin{aligned}
& \int_{\operatorname{Mat}\left(n, \mathbb{Q}_{p}\right)} \gamma(z)^{-t} d \operatorname{vol}(z)=\operatorname{vol}(K) \int_{G}|\operatorname{det}(z)|^{n} \gamma(z)^{-t} d \chi(z)= \\
&=\operatorname{vol}(K) \int_{G}|\operatorname{det}(z)|^{-t+n} \operatorname{vol}\left(z \mathbb{O}_{p}^{n} \cap \mathbb{O}_{p}^{n}\right)^{t} d \chi(z)= \\
&=\operatorname{vol}(K) \int_{G} \operatorname{vol}\left(z \mathbb{O}_{p}^{n}\right)^{-t+n} \operatorname{vol}\left(z \mathbb{O}_{p}^{n} \cap \mathbb{O}_{p}^{n}\right)^{t} d \chi(z) .
\end{aligned}
$$

The integrand is constant on each coset $z K \subset G$, by the invariance of Haar measure, we have $\chi(z K)=1$. Therefore we come to a summation over $G / K=$ Lat $(n)$ :

$$
\operatorname{vol}(K) \sum_{Q \in \operatorname{Lat}(n)} \operatorname{vol}(Q)^{-t+n} \operatorname{vol}\left(Q \cap \mathbb{O}_{p}^{n}\right)^{t} .
$$

This expression is a special case of the 'beta-function of Bruhat-Tits building' evaluated in [9], Theorem 2.1. In notation of [9], we set

$$
\alpha_{1}=\cdots=\alpha_{n}=t \quad \beta_{1}=\cdots=\beta_{n}=-t+n
$$

and get

$$
\operatorname{vol}(K) \prod_{j=1}^{n} \frac{1-p^{-t+n-j}}{\left(1-p^{-t+n+j-1}\right)\left(1-p^{-j}\right)}=\prod_{j=1}^{n} \frac{1-p^{-t+n-j}}{\left(1-p^{-t+n+j-1}\right)}
$$

\section{Proofs. Transformation of measures}

Here we prove Theorem 1.5.

3.1. Formula for Radon-Nikodym derivative. 1) Consider the subgroup $P \subset \operatorname{GL}\left(2 n, \mathbb{O}_{p}\right)$ consisting of matrices $\left(\begin{array}{ll}a & b \\ 0 & d\end{array}\right)$, it $\operatorname{acts}$ on $\operatorname{Mat}\left(n, \mathbb{Q}_{p}\right)$ by the transformations

$$
z \mapsto a^{-1}(b+z d)=a^{-1} b+a^{-1} z d
$$


The measure $d \operatorname{vol}(z)$ is invariant with respect to such transformations. The function $\gamma(z)^{-s-2 n}$ also is invariant. Therefore the measure $\mu_{s}^{n}$ is $P$-invariant.

2) Consider the matrix $\left(\begin{array}{ll}0 & 1 \\ 1 & 0\end{array}\right)$. The corresponding transformation is $z \mapsto$ $z^{-1}$. We must show that

$$
\gamma\left(z^{-1}\right)^{-s-2 n} d \operatorname{vol}\left(z^{-1}\right)=|\operatorname{det}(z)|^{s} \gamma(z)^{-s-2 n} d \operatorname{vol}(z) .
$$

First

$$
d \operatorname{vol}\left(z^{-1}\right)=|\operatorname{det}(z)|^{-2 n} d \operatorname{vol}(z),
$$

this is equivalent to the formula (2.5) for the Haar measure. On the other hand,

$$
\gamma\left(z^{-1}\right)^{-s-2 n}=\gamma(z)^{-s-2 n}|\operatorname{det} z|^{s+2 n},
$$

and we get (3.1).

3) It can be easily shown that $\operatorname{GL}\left(2 n, \mathbb{O}_{p}\right)$ is generated by subgroup $P$ and $\left(\begin{array}{ll}0 & 1 \\ 1 & 0\end{array}\right)$. Denote

$$
z * g:=(a+z c)^{-1}(b+z d) .
$$

The Radon-Nikodym derivative

$$
c(g, z):=\frac{d \mu_{s}^{n}(z * g)}{d \mu_{s}^{n}(z)} .
$$

satisfies the chain rule

$$
c\left(g_{1} g_{2}, z\right)=c\left(g_{1}, z\right) c\left(g_{2}, z * g_{1}\right) .
$$

On the other hand, the expression

$$
\widetilde{c}(g, z):=|\operatorname{det}(a+z c)|^{s}
$$

also satisfies the chain rule. Since $c(h, z)=\widetilde{c}(h, z)$ for generators of $G$, they coincide everywhere.

3.2. Uniqueness of the measure. Consider a measure $\nu$ satisfying equation (1.6). First, we consider matrices $\left(\begin{array}{ll}1 & b \\ 0 & 1\end{array}\right)$ and get that $\nu$ is invariant with respect to translations

$$
z \mapsto z+b, \quad b \in \operatorname{Mat}\left(n, \mathbb{O}_{p}\right) .
$$

Therefore $\nu$ has the form

$$
d \nu(z)=f(z) d \mu_{s}^{n}(z)
$$

where $f(z)$ is a locally constant function. Evidently, $f(z)$ is $\operatorname{GL}\left(2 n, \mathbb{O}_{p}\right)$-invariant. Since the action of $\mathrm{GL}\left(2 n, \mathbb{O}_{p}\right)$ on the Grassmannian is transitive, we get that $f(z)$ is constant. 


\section{Proofs. Projective limits}

4.1. Proof of Theorem 1.3. It is sufficient to show that the $\Pi$-pushforward $\nu$ of $\mu_{s}^{n+1}$ satisfies the quasiinvariance property (1.6). It is sufficient to verify this property for generators of $\operatorname{GL}\left(2 n, \mathbb{O}_{p}^{n}\right)$ :

$$
g_{1}=\left(\begin{array}{cc}
a & 0 \\
0 & d
\end{array}\right), \quad g_{2}=\left(\begin{array}{ll}
1 & b \\
0 & 1
\end{array}\right), \quad g_{3}=\left(\begin{array}{ll}
0 & 1 \\
1 & 0
\end{array}\right) .
$$

We consider the corresponding elements of $\operatorname{GL}\left(2(n+1), \mathbb{O}_{p}\right)$ :

$$
\widetilde{g}_{1}=\left(\begin{array}{cccc}
a & 0 & 0 & 0 \\
0 & 1 & 0 & 0 \\
0 & 0 & d & 0 \\
0 & 0 & 0 & 1
\end{array}\right), \quad \widetilde{g}_{2}=\left(\begin{array}{cccc}
1 & 0 & b & 0 \\
0 & 1 & 0 & 0 \\
0 & 0 & 1 & 0 \\
0 & 0 & 0 & 1
\end{array}\right), \quad \widetilde{g}_{3}=\left(\begin{array}{cccc}
0 & 0 & 1 & 0 \\
0 & 1 & 0 & 0 \\
1 & 0 & 0 & 0 \\
0 & 0 & 0 & 1
\end{array}\right)
$$

(sizes of the matrices are $n+1+n+1$ ). We have

$$
\begin{aligned}
& \widetilde{g}_{1}:\left(\begin{array}{ll}
z_{11} & z_{12} \\
z_{21} & z_{22}
\end{array}\right) \mapsto\left(\begin{array}{ll}
a & 0 \\
0 & 1
\end{array}\right){ }^{-1}\left(\begin{array}{ll}
z_{11} & z_{12} \\
z_{21} & z_{22}
\end{array}\right)\left(\begin{array}{ll}
d & 0 \\
0 & 1
\end{array}\right)=\left(\begin{array}{cc}
a^{-1} z_{11} d & * \\
* & *
\end{array}\right) ; \quad(4.1) \\
& \widetilde{g}_{2}:\left(\begin{array}{ll}
z_{11} & z_{12} \\
z_{21} & z_{22}
\end{array}\right) \mapsto\left(\begin{array}{ll}
b & 0 \\
0 & 1
\end{array}\right)+\left(\begin{array}{ll}
z_{11} & z_{12} \\
z_{21} & z_{22}
\end{array}\right)=\left(\begin{array}{cc}
b+z_{11} & * \\
* & *
\end{array}\right) ; \\
& \widetilde{g}_{3}:\left(\begin{array}{ll}
z_{11} & z_{12} \\
z_{21} & z_{22}
\end{array}\right) \mapsto \\
& \mapsto\left[\left(\begin{array}{ll}
0 & 0 \\
0 & 1
\end{array}\right)+\left(\begin{array}{ll}
1 & 0 \\
0 & 0
\end{array}\right)\left(\begin{array}{ll}
z_{11} & z_{12} \\
z_{21} & z_{22}
\end{array}\right)\right]^{-1}\left[\left(\begin{array}{ll}
1 & 0 \\
0 & 0
\end{array}\right)+\left(\begin{array}{cc}
0 & 0 \\
0 & 1
\end{array}\right)\left(\begin{array}{cc}
z_{11} & z_{12} \\
z_{21} & z_{22}
\end{array}\right)\right]= \\
& =\left(\begin{array}{cc}
z_{11} & 0 \\
z_{21} & 1
\end{array}\right)
\end{aligned}
$$

Thus we get

$$
\Pi\left(z * \widetilde{g}_{j}\right)=(\Pi z) * g_{j}
$$

In the first two cases the Radon-Nikodym derivative is 1 , in the last case $\left|\operatorname{det} z_{11}\right|^{s}$. Thus,

$$
\frac{d \mu_{s}^{n+1}\left(z * \widetilde{g}_{j}\right)}{d \mu_{s}^{n+1}(z)}=\frac{d \mu_{s}^{n}\left((\Pi z) * g_{j}\right)}{d \mu_{s}^{n}(\Pi z)} .
$$

This implies desired property of the П-pushforward of $\mu_{s}^{n+1}$.

4.2. An abstract lemma. Let $\left(\Omega_{j}, \mu_{j}\right)$ be a sequence of Lebesgue measure spaces with probability measures. Let $\Pi_{k}^{j}: \Omega_{j} \rightarrow \Omega_{k}$, where $k<j$, be maps such that $\Pi_{l}^{k} \Pi_{k}^{j}=\Pi_{l}^{j}$ and the $\Pi_{k}^{j}$-pushforward of $\mu_{j}$ is $\mu_{k}$. Denote by $\left(\Omega_{\infty}, \mu_{\infty}\right)$ the projective limit of the chain

$$
\ldots \longleftarrow \Omega_{k} \longleftarrow \Omega_{k+1} \longleftarrow \ldots
$$


Let $G$ be a group. Let for any $g \in G$ there exists $j$ such that for all $k \geqslant j$ there is a transformation $g_{[k]}: \Omega_{k} \rightarrow \Omega_{k}$ leaving the measure $\mu_{j}$ quasiinvariant and for $l>k>j$ we have (a.s.)

$$
\Pi_{k}^{l}\left(g_{[l]}(\omega)\right)=g_{[k]}\left(\Pi_{k}^{l}(\omega)\right), \quad \omega \in \Omega_{l},
$$

and (a.s.)

$$
\left(g_{[k]}\right)^{\prime}\left(\Pi_{[k]}^{l} \omega\right)=\left(g_{[l]}\right)^{\prime}(\omega), \quad \omega \in \Omega_{l} .
$$

Let also for any $g, h \in G$ for sufficiently large $m$

$$
(g h)_{[m]}=g_{[m]} h_{[m]} .
$$

Lemma 4.1 Under these conditions there is action of $G$ on $\Omega_{\infty}$ by transformations $g_{[\infty]}$ leaving the measure $\mu_{\infty}$ quasiinvariant, they are determined by

$$
\Pi_{k}^{\infty}\left(g_{[\infty]}(\omega)\right)=g_{[k]}\left(\Pi_{k}^{\infty}(\omega), \quad \omega \in \Omega_{\infty},\right.
$$

and

$$
\left(g_{[k]}\right)^{\prime}\left(\Pi_{[k]}^{\infty} \omega\right)=\left(g_{[\infty]}\right)^{\prime}(\omega), \quad \omega \in \Omega_{\infty} .
$$

This is straightforward.

Proposition 1.4 is an immediate corollary of the lemma.

4.3. Action of $\overline{\overline{\mathrm{GL}}}\left(2 \infty, \mathbb{O}_{p}\right)$. We wish to reduce Theorem 1.5 to Lemma 4.1. Now $\Omega_{k}:=\operatorname{Mat}\left(k, \mathbb{Q}_{p}\right)$, projections $\Omega_{l} \rightarrow \Omega_{k}$ are cutting of left upper $k \times k$ corner. We must construct transformations $g_{[k]}: \operatorname{Mat}\left(k, \mathbb{Q}_{p}\right) \rightarrow \operatorname{Mat}\left(k, \mathbb{Q}_{p}\right)$.

Fix $g \in \overline{\mathrm{GL}}\left(2 \infty, \mathbb{O}_{p}\right)$. Choose a sufficiently large $k$ and represent $g=\left(\begin{array}{ll}a & b \\ c & d\end{array}\right)$ in the form

$$
g=\left(\begin{array}{cccc}
a_{11} & 0 & b_{11} & b_{12} \\
a_{21} & a_{22} & b_{21} & b_{22} \\
c_{11} & 0 & d_{11} & d_{12} \\
0 & 0 & 0 & d_{22}
\end{array}\right)=\left(\begin{array}{cccc}
a_{11}^{(k)} & 0 & b_{11}^{(k)} & b_{12}^{(k)} \\
a_{21}^{(k)} & a_{22}^{(k)} & b_{21}^{(k)} & b_{22}^{(k)} \\
c_{11}^{(k)} & 0 & d_{11}^{(k)} & d_{12}^{(k)} \\
0 & 0 & 0 & d_{22}^{(k)}
\end{array}\right)
$$

(below we sometimes omit upper index ${ }^{(k)}$ ). We formally calculate

$$
\begin{gathered}
(a+z c)^{-1}(b+z d)= \\
{\left[\left(\begin{array}{cc}
a_{11} & 0 \\
a_{21} & a_{22}
\end{array}\right)+\left(\begin{array}{ll}
z_{11} & z_{12} \\
z_{21} & z_{22}
\end{array}\right)\left(\begin{array}{cc}
c_{11} & 0 \\
0 & 0
\end{array}\right)\right]^{-1}\left[\left(\begin{array}{ll}
b_{11} & b_{12} \\
b_{21} & b_{22}
\end{array}\right)+\left(\begin{array}{cc}
z_{11} & z_{12} \\
z_{21} & z_{22}
\end{array}\right)\left(\begin{array}{cc}
d_{11} & d_{12} \\
0 & d_{22}
\end{array}\right)\right]} \\
=\left(\begin{array}{cc}
\left(a_{11}+z_{11} c_{11}\right)^{-1} & 0 \\
* & *
\end{array}\right)\left(\begin{array}{cc}
b_{11}+z_{11} d_{11} & * \\
* & *
\end{array}\right)= \\
=\left(\begin{array}{cc}
\left(a_{11}^{(k)}+z_{11}^{(k)} c_{11}^{(k)}\right)^{-1}\left(b_{11}^{(k)}+z_{11}^{(k)} d_{11}^{(k)}\right) & * \\
* & *
\end{array}\right) .
\end{gathered}
$$


We observe that for sufficiently large $k$ the $k \times k$ left upper corner of $(a+$ $z c)^{-1}(b+z d)$ depends only on the $z_{11}^{(k)}$. Now we assign the transformation

$$
g_{[k]}: u \mapsto\left(a_{11}^{(k)}+u c_{11}^{(k)}\right)^{-1}\left(b_{11}^{(k)}+u d_{11}^{(k)}\right)
$$

of $\operatorname{Mat}\left(k, \mathbb{Q}_{p}\right)$. The same calculation shows the compatibility (4.3).

Next, let us write formally the Radon-Nikodym derivative of our transformation is

$$
\left|\operatorname{det}\left(\begin{array}{cc}
a_{11}+z_{11} c_{11} & 0 \\
a_{21}+z_{21} c_{11} & a_{22}
\end{array}\right)\right|^{s}=\left|\operatorname{det}\left(a_{11}+z_{11} c_{11}\right)\right|^{s}\left|\operatorname{det} a_{22}\right|^{s} .
$$

But $a_{22}$ is lower triangular and we can set $\left|\operatorname{det} a_{22}\right|=1$.

Note that the expression(4.5) coincides with the Radon-Nikodym derivative of $g_{[k]}$ and does not change under a pass $k \rightarrow k+1$. Therefore we have compatibility (4.3).

4.4. Proof of Proposition 1.6. For fixed $g=\left(\begin{array}{ll}a & b \\ c & d\end{array}\right) \in \overline{\mathrm{GL}}\left(2 n, \mathbb{Q}_{p}\right)$, The following expression

$$
f(z)=\operatorname{det}\left(\begin{array}{cc}
a_{11}+z_{11} c_{11} & 0 \\
a_{21}+z_{21} c_{11} & a_{22}
\end{array}\right):=\operatorname{det}\left(a_{11}+z_{11} c_{11}\right)
$$

is a well-defined function on $\operatorname{Mat}\left(\infty, \mathbb{Q}_{p}\right)$.

Also note that this function satisfies the chain rule (3.3). Therefore formula (1.12) determines a representation of the group $\overline{\mathrm{GL}}(2 \infty, \mathbb{O})$.

\section{References}

[1] Borodin, A.; Olshanski, G. Harmonic analysis on the infinite-dimensional unitary group and determinantal point processes. Ann. of Math. (2) 161 (2005), 1319-1422

[2] Hua Loo Keng, Harmonic analysis of functions of several complex variables in the classical domains. Science Press, Peking 1958 (in Chinese), Izdat. Inostr. Lit., Moscow 1959 (in Russian), Amer. Math. Soc., Providence, R.I., 1963.

[3] Kerov, S., Olshanski, G., Vershik, A. Harmonic analysis on the infinite symmetric group. Invent. Math. 158, No. 3, 551-642 (2004)

[4] Macdonald, I.G. Symmetric functions and Hall polynomials. Second edition, Clarendon Press, 1996

[5] Nazarov, M. Oscillator semigroup over a non-Archimedean field. J. Funct. Anal. 128 (1995), no. 2, 384-438 
[6] Nazarov, M.; Neretin, Yu.; Olshanskii, G. Semi-groupes engendrés par la représentation de Weil du groupe symplectique de dimension infinie. C. R. Acad. Sci. Paris Sèr. I Math. 309 (1989), no. 7, 443-446.

[7] Neretin, Yu. A. Categories of symmetries and infinite-dimensional groups. Oxford University Press, New York, 1996.

[8] Neretin, Yu. A. Hua-type integrals over unitary groups and over projective limits of unitary groups. Duke Math. J. 114 (2002), no. 2, 239-266.

[9] Neretin, Yu. A. The beta function of the Bruhat-Tits building and the deformation of the space $l^{2}$ on the set of p-adic lattices. Sb. Math. 194 (2003), no. 11-12, 1775-1805

[10] Neretin, Yu. A. Lectures on Gaussian integral operators and classical groups. European Mathematical Society (EMS), Zürich, 2011

[11] Neretin, Yu. A. Infinite-dimensional p-adic groups, semigroups of double cosets, and inner functions on Bruhat-Tits builldings. arXiv:1108.4873

[12] Olshanski, G. I. Unitary representations of infinite-dimensional pairs $(G, K)$ and the formalism of $R$. Howe. Soviet Math. Dokl. 27 (1982), no. 2, 290-294

[13] Olshanski, G.I., Unitary representations of $(G, K)$-pairs connected with the infinite symmetric group $S(\infty)$. Leningr. Math. J. 1, No.4, 983-1014 (1990).

[14] Olshanski, G.I. Unitary representations of infinite dimensional pairs $(G, K)$ and the formalism of $R$. Howe. In Representation of Lie groups and related topics, Adv. Stud. Contemp. Math. 7, 269-463 (1990).

[15] Olshanski, G.I. The problem of harmonic analysis on the infinitedimensional unitary group. J. Funct. Anal., 205 (2003), 464-524.

[16] Olshanski, G. I. On semigroups related to infinite-dimensional groups. In: Topics in representation theory (A. A. Kirillov, ed.). Advances in Soviet Math., vol. 2. Amer. Math. Soc., Providence, R.I., 1991, 67-101.

[17] Ørsted, B. A model for an interacting quantum field. J. Funct. Anal. 36 (1980), no. $1,53-71$.

[18] Pickrell, D. Measures on infinite-dimensional Grassmann manifolds, J. Funct. Anal. 70, No. 2 (1987), 323-356

[19] Pickrell, D. Separable representations for automorphism groups of infinite symmetric spaces. J. Funct. Anal. 90 (1990), no. 1, 1-26.

[20] Shiryaev, A. N. Probability, Springer, 1984

[21] Vershik, A. M.; Kerov, S. V. Characters and factor-representations of the infinite unitary group. Soviet Math. Dokl. 26, 570-574 (1983). 
[22] Vershik, A. M.; Kerov, S. V. Asymptotic theory of the characters of a symmetric group. Functional Anal. Appl. 15 (1981), no. 4, 246-255 (1982).

[23] Vershik, A. M.; Kerov, S. V. Four drafts on the representation theory of the group of infinite matrices over a finite field. J. Math. Sci. (N. Y.) 147 (2007), no. 6, 7129-7144

[24] Zelenov, E. I. A p-adic infinite-dimensional symplectic group. Russian Acad. Sci. Izv. Math. 43 (1994), no. 3, 421-441

Math.Dept., University of Vienna,

Nordbergstrasse, 15, Vienna, Austria

\&

Institute for Theoretical and Experimental Physics,

Bolshaya Cheremushkinskaya, 25, Moscow 117259, Russia

$\&$

Mech.Math.Dept., Moscow State University,

Vorob'evy Gory, Moscow

e-mail: neretin(at) mccme.ru

URL : www . mat. univie.ac.at/ neretin

wwwth. itep.ru/ neretin 